\title{
A hybrid approach to molecular continuum processes combining Gaussian basis functions and the discrete variable representation
}

\author{
T. N. Rescigno, ${ }^{1}$ D. A. Horner, ${ }^{2}$ F. L. Yip, ${ }^{2}$ and C. W. McCurdy ${ }^{3,1}$ \\ ${ }^{1}$ Lawrence Berkeley National Laboratory, Chemical Sciences, Berkeley, CA 94720 \\ ${ }^{2}$ Department of Chemistry, University of California, Berkeley, CA 94720 \\ ${ }^{3}$ Departments of Applied Science and Chemistry, University of California, Davis, CA 95616
}

\begin{abstract}
Gaussian basis functons, routinely employed in molecular electronic structure calculations, can be combined with numerical grid-based functions in a discrete variable representation to provide an efficient method for computing molecular continuum wave functions. This approach, combined with exterior complex scaling, obviates the need for slowly convergent single-center expansions, and allows one to study a variety of electron-molecule collision problems. The method is illustrated by computation of various bound and continuum properties of $\mathrm{H}_{2}^{+}$.
\end{abstract}

\section{INTRODUCTION}

The theoretical treatment of electron-impact ionization and double photoionization of small atomic and molecular species has, in recent years, become an important and active area of research. For the simplest atomic three-body Coulomb systems [1, 2], significant advances in both theory and computation have been made. It is now possible to carry out a complete firstprinciples "reduction to computation" of virtually all aspects of the problem, including the most detailed fully differential cross sections, for both electron-impact ionization " $(\mathrm{e}, 2 \mathrm{e})$ " and double photoionization " $(\gamma, 2 \mathrm{e})$ ". Grid-based numerical methods employing exterior complex scaling (ECS) have played a key role in this development and have been successful in treating problems characterized by the presence of one or two continuum electrons in the final state [3]. In general, complex scaling avoids the explicit enforcement of asymptotic boundary conditions by imposing a transformation on the electron coordinates that causes outgoing waves to decay exponentially [4]. Exterior complex scaling [5, 6] also simplifies the imposition of asymptotic boundary conditions through the use of complex coordinates, but by scaling electron coordinates only beyond the radius of a hypersphere, it allows physical quantities to be extracted from a region of space where all coordinates are real. $\mathrm{Nu}-$ merical grid-based treatments such as finite difference schemes [2], finite element methods [7, 8], the discrete variable representation (DVR) coupled with finite elements [9], and most recently, B-spline [10, 11] examples, have been successful in incorporating exterior complex scaling for the solution of scattering problems, providing converged solutions that are insensitive to the details of exterior complex scaling parameters.

While the extension of current methods to simple molecular targets is conceptually straightforward, the practical consequences of treating a non-spherically symmetric target present formidable computational challenges. By choosing product basis functions for each electron of the form

$$
\chi_{i l m}(\mathbf{r})=f_{i}(r) Y_{l, m}(\hat{\mathbf{r}}),
$$

composed of a radial part $f_{i}(r)$ and spherical harmonics $Y_{l, m}(\theta, \phi)$, to represent the angular coordinates, then ECS can be simply applied to the radial coordinates to produce outgoing wave solutions that decay exponentially beyond some fixed radial point $r_{\text {ecs }}$. A basis of radial functions with compact support, such as B-splines [12] or DVR position eigenfunctions [13], is particularly useful for describing electronic coordinates at large $r$, thus allowing for a large region of space to be efficiently described almost completely. In addition to being well suited for ECS, that is, without acquiring physical consequences sensitive to the details of complex scaling [10], the use of such functions facilitate the computation of matrix elements through simple numerical formulas and produce sparse or structured matrices that can easily be computed and diagonalized. An atomic product basis of the type given in Eq. (1) could also be used, in conjunction with an expansion about a single-center, to treat molecular problems, but the lack of spherical symmetry results in coupling between the various angular momentum channels that is not present in the atomic case. The single-center approach has in fact been employed, in conjuction with an ECS implementation using B-splines, in a first-principles study of double photoionization of molecular hydrogen [14]. While such an approach is viable for the simplest molecular targets such as $\mathrm{H}_{2}$ or $\mathrm{D}_{2}$, it would be difficult to generalize to heavier systems, since single-center expansions become increasingly difficult to conve7rge as the nuclear charge increases beyond one and would require many partial waves to achieve accurate results.

In contrast to numerical grid schemes, analytic basis functions have been extensively applied to molecular problems, particularly to bound-state molecular electronic structure problems. Gaussian basis functions are ubiquitous in computational quantum chemistry, since matrix elements involving Gaussians centered on diffrent nuclei can be evaluated in terms of known analytic functions. The inherent local nature of Gaussian functions also means that they are not, by themselves, well-suited for scattering calculations where electrons must be well described at distances far from nuclear centers. The inclusion of increasingly diffuse Gaussians or expansively 

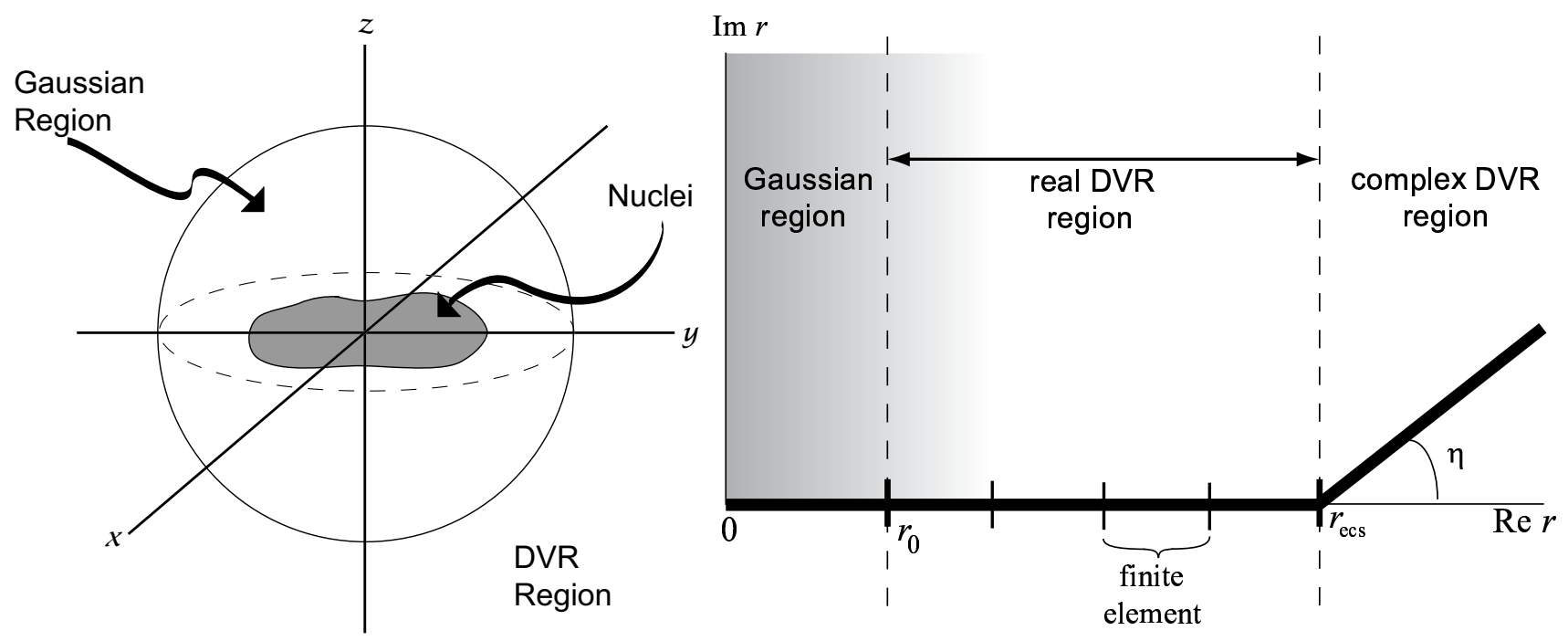

FIG. 1: Description of areas of physical space where different basis representations of electronic coordinates are best suited. In a mixed-basis composed of Gaussians and DVR functions, a radial interface $r_{0}$ specifies the linkage between the two constituent bases.

large basis sets as a remedy to their locality eventually fails due to the development of linear dependencies within the Gaussian basis. Furthermore, in connection with exterior complex scaling, Gaussian functions, like other analytic basis functions, require a cumbersome "smoothed" exterior scaling treatment that can produce unwanted physical consequences [15-17].

It is our intent here to demonstrate that Gaussian basis functions, which are ideally suited to describing the multi-center behavior of molecular wave functions over a restriced region of space, can be combined with exterior scaled finite-element/DVR functions to provide a hybrid basis that is well suited for applications to molecular scattering problems, including $(e, 2 e)$ and $(\gamma, 2 e)$ problems. Moreover, the combination of numerical grid-based approaches with traditional Gaussian basis expansions allows one to make a connection with standard quantum chemistry descriptions of many-electron target states. Such connections were central to the development of modern variational methods used in electron-polyatomic molecule scattering calculations and are a key motivating factor in the present work. The formulation of a proper linearly independent aggregate basis requires consideration of the coupling bewteen the two component bases and a practical scheme for evaluating the requisite matrix elements in the hybrid basis. The following section describes the hybrid basis and outlines the construction of one-electron operators. In Section III we provide some illustrative examples involving the hydrogen molecular ion $\mathrm{H}_{2}^{+}$. We conclude with a brief discussion.

\section{CONSTRUCTION OF A HYBRID GAUSSIAN-DVR BASIS}

The mixed Gaussian-DVR basis is defined by the regions of space where either Gaussian basis functions or DVR basis functions are better suited to compute physical quantities. We therefore begin by partitioning space into an inner region $0 \leq r<r_{0}$, which contains the nuclei, and an outer region which extends to some large distance $r_{\max }$. For scattering problems, the outer region is further divided into an intermediate region $r_{0} \leq r<r_{\text {ecs }}$ and an asymptotic region $r_{\text {ecs }} \leq r<r_{\text {max }}$. This spatial partitioning is depicted in Fig. 1. Electron radial coordinates will be complex- scaled only in the asymptotic region under the ECS transformation,

$$
r \rightarrow \begin{cases}r & r<r_{\text {ecs }} \\ r_{\text {ecs }}+\left(r-r_{\text {ecs }}\right) e^{i \eta} & r \geq r_{\text {ecs }}\end{cases}
$$

We must choose $r_{\text {ecs }}$ large enough that the interaction potentials can be safely truncated beyond that point. Since the DVR basis functions have compact support and do not extend inward of $r_{0}$, the inner region will be spaned only by Gaussians. The Gaussians, on the other hand, can extend into the intermediate region, but are assumed to be negligible in the asymptotic region. This requires that the Gaussian basis and DVR grid be chosen to ensure all Gaussian functions are effectively zero beyond $r_{\text {ecs }}$. For small molecules, this is generally not a problem since $r_{\text {ecs }}$ is typically located tens of bohr away from the nuclei. The location of the interface $r_{0}$ which marks the beginning of the DVR region depends on several factors. Generally, these include the fixed nuclear geometry and the nature (i. e. , radial extent) of the Gaussian basis. Before discussing the factors which determine the optimal 
location of $r_{0}$ and the operational interplay between the Gaussian and DVR basis functions, it is appropriate to elaborate on the dominant basis functions of the intervals separated by $r_{0}$ in Fig. 1.

Little need be said here about the choice of Gaussian functions as a basis for expanding molecular orbitals. Since the independent proposal of Boys [18] and McWeeny [19], the use of Gaussian basis functions has pervaded atomic and molecular electronic structure theory [20]. Although atomic orbitals are better described by Slater-type orbitals (STOs), Gaussian basis functions (GTOs) have distinct computational advantages over STOs in molecular calculations, benefiting from the fact that the product of two Gaussians on different centers yields another Gaussian centered along the line connecting the two. This fact results in computational efficiency relative to STOs by reducing the evaluation of two-electron Coulomb repulsion matrix elements for twoelectron operators to calculations involving at most twocenter integrals. The most common type of Gaussians, which we also employ here, are Cartesian Gaussian functions of the form

$G_{\Gamma}(\mathbf{r})=N\left(x-X_{\Gamma}\right)^{l_{\Gamma}}\left(y-Y_{\Gamma}\right)^{m_{\Gamma}}\left(z-Z_{\Gamma}\right)^{n_{\Gamma}} e^{-\alpha_{\Gamma}\left|\mathbf{r}-\mathbf{R}_{\Gamma}\right|^{2}}$,

where $N$ is a normalization constant and $\left(X_{\Gamma}, Y_{\Gamma}, Z_{\Gamma}\right)$ are the Cartesian components of the center $\mathbf{R}_{\Gamma}$. These "primitive" Gaussians are frequently employed as basis functions in fixed linear combinations or "contractions", which are chosen to model STOs for the construction of atomic or molecular orbitals [21-25].

Many one-electron matrix elements, including overlap integrals

$$
S_{\Gamma \Gamma^{\prime}}=\int_{0}^{\infty} G_{\Gamma}(\mathbf{r}) G_{\Gamma^{\prime}}(\mathbf{r}) d \mathbf{r}
$$

as well as kinetic energy and electron-nuclear attraction matrix elements,

$$
\begin{aligned}
& T_{\Gamma \Gamma^{\prime}}=-\frac{1}{2} \int_{0}^{\infty} G_{\Gamma}(\mathbf{r}) \nabla^{2} G_{\Gamma^{\prime}}(\mathbf{r}) d \mathbf{r}, \\
& V_{\Gamma \Gamma^{\prime}}=\int_{0}^{\infty} G_{\Gamma}(\mathbf{r}) \frac{1}{|\mathbf{r}-\mathbf{R}|} G_{\Gamma^{\prime}}(\mathbf{r}) d \mathbf{r}
\end{aligned}
$$

can be expressed in closed form in terms of well known analytic functions [20]. The computation of these quantities, as well as other one- and two-electron matrix elements, has been continuously refined and incorporated into numerous atomic and molecular structure software packages.

The discrete variable representation that we employ takes the analysis of Manolopoulos and Wyatt [26] as its point of departure. It combines a high order treatment of the kinetic energy operator in a polynomial basis with the advantage of providing a diagonal representation of the radial portion of any local operator, which dramatically simplifies the computation of matrix elements of the potential. On the interval $[a, b]$ one can define normalized DVR basis functions in terms of Lagrange interpolating polynomials as

$$
f_{i}(r)=\left\{\begin{array}{ll}
w_{i}^{-1 / 2} \prod_{j \neq i} \frac{r-r_{j}}{r_{i}-r_{j}} & a \leq r \leq b \\
0 & \text { otherwise }
\end{array} .\right.
$$

The mesh points $\left[r_{i}\right]$ and weights $\left[w_{i}\right]$ are derived from a Gauss-Lobatto quardature. Gauss-Lobatto quadrature is similar to the more familiar Gauss-Legendre quadrature, both of which approximate integrals as,

$$
\int_{a}^{b} F(r) d r \approx \sum_{i=1}^{n} F\left(r_{i}\right) w_{i}
$$

In Gauss-Lobatto quadrature, two of the points are constrained to coincide with the end points, which means that Eq.(8) can be made exact when $F(r)$ is a polynomial of degree $\leq 2 n-1$.

The DVR functions have the property, when evaluated at the mesh points, that

$$
f_{i}\left(r_{j}\right)=\delta_{i, j} / \sqrt{w_{i}}
$$

and they are thus orthonormal under Gauss-Lobatto integration,

$$
\int_{a}^{b} f_{i}(r) f_{j}(r) d r \approx \sum_{k=1}^{n} f_{i}\left(r_{k}\right) f_{j}\left(r_{k}\right) w_{k}=\delta_{i, j} .
$$

It also follows from Eq. (9) that, under Gauss-Lobatto integration, the DVR functions give a diagonal representation of any local radial operator:

$$
\begin{aligned}
\int_{a}^{b} f_{i}(r) V(r) f_{j}(r) d r & \approx \sum_{k=1}^{n} f_{i}\left(r_{k}\right) V\left(r_{k}\right) f_{j}\left(r_{k}\right) w_{k} \\
& =\delta_{i, j} V\left(r_{i}\right) .
\end{aligned}
$$

Note that, while the Gauss-Lobatto quadrature rules only require the evaluation of operators at the mesh points, the DVR functions, through Eq. (7), have an underlying continuous representation that can be used to evaluate the wave function at any value of $r$.

Since Gauss-Lobatto quadrature explictly includes the end points as quadrature points, it is possible to combine this particular variety of DVR with the finite-element method by imposing continuity conditions at element boundaries. In the FEM-DVR approach [9] we divide the ECS contour for the radial coordinate of each electron into one-dimensional finite elements with one of the boundaries coinciding with the point $r_{\text {ecs }}$ where the real and complex parts of the contour join. In each element we use the same order $n$ Gauss-Lobatto quadrature. In the proposed hybrid method, the first radial element begins, not at the origin, but at the point $r_{0}$. The boundary condition that the wave function vanish at the end of the grid is imposed by simply excluding the DVR function associated with the last point $r_{\max }$. Similarly, we exclude the DVR function associated with the point $r_{0}$ to insure continuity of the overall basis across that point. This does 
not imply an unphysical constraint on the wave function, since the Gaussian portion of the basis does not vanish at $r_{0}$. Further details about the FEM-DVR procedure can be found in refs. [9] and [3].

The hybrid basis, then, consists of a set of Gaussian functions which span the inner region centered on the molecule and extends beyond $r_{0}$, but not beyond $r_{\mathrm{ecs}}$, along with a set of orthonormal DVR functions, which begin at $r_{0}$, are real-valued out to $r_{\mathrm{ecs}}$ and complex from $r_{\text {ecs }}$ to $r_{\max }$. These DVR functions are then combined with spherical harmonics to complete the basis. Matrix elements involving only Gaussian functions can be easily calculated using known analytic expressions, while matrix elements involving DVR functions or Gaussians and DVR functions are computed numerically. We found that the radial portions of all of the DVR and most of the mixed Gaussian/DVR matrix elements were adequately approximated using Gauss-Lobatto quadrature. The sole exception was found to be the overlap integrals between Gaussians and DVR functions which we found to be needed to very high accuracy. The radial parts of these integrals were therefore computed using high-order Gauss-Legendre quadrature. The angular parts of all numerically computed matrix elements were performed to machine accuracy using high-order GaussMarkov quadratures tabulated by Lebedev and Laikov [27].

\section{ILLUSTRATIVE EXAMPLES: BOUND AND CONTINUUM STATES OF $\mathrm{H}_{2}^{+}$}

\section{A. Bound States}

To illustrate the power of the hybrid representation discussed above, we present the results of several calculations on the simplest one-electron molecule, $\mathrm{H}_{2}^{+}$. For these calculations, the coordinate origin was placed at the midpoint of the molecular axis with the nuclei situated along the $z$-axis. The Born-Oppenheimer electronic Hamiltonian for $\mathrm{H}_{2}^{+}$in atomic units $\left(\hbar=e=m_{e}=1\right)$ is

$$
H=-\frac{1}{2} \nabla^{2}-\frac{1}{|\mathbf{r}-\mathbf{A}|}-\frac{1}{|\mathbf{r}+\mathbf{A}|}
$$

In this geometry, the azimuthal quantum number $m$ is a good quantum number and eigenstates possess a definite parity. For all problems considered, the Gaussian basis consisted of six $s$-type functions and four $p$-type functions centered on each nucleus. The $s$-type functions were simply chosen as the six functions with the largest exponents from Huzinaga's ten- $s$ expansion of the hydrogen $1 s$ function in Gaussians [21]. The orbital exponents for the $p$-type functions were chosen as 3.0, 1.5, 0.75 and 0.325 . This modest-sized basis of Gaussians is by itself incapable of giving a good description of any of the bound states of $\mathrm{H}_{2}^{+}$. The calculations were all carried out at the equilibrium internuclear distance, $R=2.0$ bohr.
TABLE I: Energies of the ${ }^{2} \Sigma_{g, u}$ states of $\mathrm{H}_{2}^{+}$at an internuclear distance of $R=2.0 \mathrm{bohr}$.

\begin{tabular}{ccc}
\hline \hline State & \multicolumn{2}{c}{ Energy (a. u.) } \\
\cline { 2 - 3 } & Hybrid basis & Reference [28] \\
\hline $1 \mathrm{~s} \sigma_{g}$ & -0.602619 & -0.602634 \\
$2 \mathrm{p} \sigma_{u}$ & -0.167523 & -0.167534 \\
$2 \mathrm{~s} \sigma_{g}$ & 0.139140 & 0.139135 \\
$3 \mathrm{p} \sigma_{u}$ & 0.244589 & 0.244587 \\
$3 \mathrm{~d} \sigma_{g}$ & 0.264230 & 0.264222 \\
$3 \mathrm{~s} \sigma_{g}$ & 0.322321 & 0.322319 \\
$4 \mathrm{p} \sigma_{u}$ & 0.362686 & 0.362687 \\
$4 \mathrm{~d} \sigma_{g}$ & 0.369210 & 0.369208 \\
$4 \mathrm{f} \sigma_{u}$ & 0.373355 & 0.373356 \\
$5 \mathrm{f} \sigma_{u}$ & 0.419349 & 0.419156 \\
$5 \mathrm{~g} \sigma_{g}$ & 0.419664 & 0.419627 \\
$6 \mathrm{~h} \sigma_{u}$ & 0.445904 & 0.444329 \\
\hline \hline
\end{tabular}

We begin with calculations on the first few bound eigenstates of ${ }^{2} \Sigma_{g}$ and ${ }^{2} \Sigma_{u}$ symmetry. For these calculations, a basis of real DVR functions, combined with spherical harmonics up to $l=7$ were generated using 17th order Lobatto quadrature in five real finite elements of length 5.0 bohr beginning at $r_{0}=1.1$ bohr. The results for gerade $(l=0,2,4$, and 6$)$ and ungerade $(l=1,3,5$, and 7 ) configurations are displayed in Table I, along with the accurate values given by Madison and Peek [28]. The small differences seen between the two sets of values can be attributed, not to errors in the DVR portion of the basis, but to the modest size of the Gaussians basis used here and consequent small errors in the wave functions in the interior regions near the nuclei. Indeed, addition of more partial wave DVR functions did not change the results to the number of figures shown.

Further evidence about convergence of the DVR expansion in the hybrid basis is provided in Table II, which shows convergence in $l$ for the lowest eignvalue in the hybrid basis, as well as in a pure single-center, DVR-only radial basis. With the hybrid basis, convergence to six significant figures is obtained with $l_{\max }=8$, while the single-center basis results, with $l_{\max }=12$, are still varying in the fourth figure.

\section{B. Continuum States}

The continuum states of the $\mathrm{H}_{2}^{+}$ion are solutions of the equation:

$$
\left[H-\frac{k^{2}}{2}\right] \Phi^{(+)}(\mathbf{k}, \mathbf{r})=0,
$$

where $H$ is the Hamiltonian defined in Eq. (12). To apply ECS to solve this equation, we must convert it into a driven equation for the scattered wave part of $\Phi^{(+)}$. The incoming wave part of the solution of $\Phi^{(+)}$is determined 
TABLE II: Convergence of the $1 \mathrm{~s} \sigma_{g}$ ground state of $\mathrm{H}_{2}^{+}$at $R=2.0$ bohr.

\begin{tabular}{rlr}
\hline \hline & \multicolumn{2}{c}{ Energy (a. u.) } \\
\cline { 2 - 3 } & \multicolumn{2}{c}{ Gaussian Basis } \\
\multicolumn{3}{c}{-0.601 860 } \\
$l_{\max }$ & Hybrid basis & DVR only \\
\hline 0 & -0.602430 & -0.518475 \\
2 & -0.602504 & -0.583470 \\
4 & -0.602599 & -0.596067 \\
6 & -0.602619 & -0.599501 \\
8 & -0.602620 & -0.600734 \\
10 & -0.602620 & -0.601264 \\
12 & -0.602620 & -0.601522 \\
\multicolumn{3}{|c}{ Reference [28] } \\
\hline \hline
\end{tabular}

by the long range behavior of the potential and thus is the same as that of the atomic Coulomb problem with $Z=2$. We can therefore write

$$
\Phi^{(+)}(\mathbf{k}, \mathbf{r})=\chi(\mathbf{k}, \mathbf{r})+g(r) \psi_{c}^{(+)}(\mathbf{k}, \mathbf{r}),
$$

where $\psi_{c}^{(+)}(\mathbf{k}, \mathbf{r})$ is the standard $(Z=2)$ Coulomb function, whose incoming momentum specifies the direction of $\mathbf{k}$ and $g(r)$ is a cutoff function which is zero at $r=0$ and must approach one as $r$ gets large, but is otherwise arbitrary. The Coulomb function can be expanded in partial waves as

$$
\psi_{c}^{(+)}(\mathbf{k}, \mathbf{r})=\left(\frac{2}{\pi}\right)^{1 / 2} \sum_{l, m} i^{l} e^{i \eta_{l}(k)} Y_{l, m}^{*}(\hat{\mathbf{k}}) \frac{\phi_{l, k}^{(\mathrm{c})}(r)}{k r}
$$

where $\phi_{l, k}^{(\mathrm{c})}(r)$ is the standard radial Coulomb function which goes asymptotically as $\sin [k r+(Z / k) \ln 2 k r-$ $\left.\pi l / 2+\eta_{l}(k)\right]$ with the Coulomb phase shift $\eta_{l}(k)$. We can therefore construct the function $\chi(\mathbf{k}, \mathbf{r})$ defined in Eq. (14) from the solutions of the driven equations

$$
\left(\frac{k^{2}}{2}-H\right) \frac{\chi(r)^{l_{0}, m}(\mathbf{r})}{r}=\left(H-\frac{k^{2}}{2}\right) g(r) \frac{\phi_{l_{0}, k}^{(c)}(r)}{r} Y_{l_{0}, m}(\hat{\mathbf{r}})
$$

To solve Eq. (16), we expand $\chi(r)^{l_{0}, m}(\mathbf{r})$ as

$$
\begin{aligned}
\frac{\chi(\mathbf{r})^{l_{0}, m}}{r} & =\sum_{\Gamma} c_{\Gamma}^{l_{0}, m} G_{\Gamma}(\mathbf{r})+\sum_{i, l} c_{i l}^{l_{0}, m} \frac{f_{i}(r)}{r} Y_{l, m}(\hat{\mathbf{r}}) \\
& \equiv \sum_{\Gamma} c_{\Gamma}^{l_{0}, m} G_{\Gamma}(\mathbf{r})+\sum_{l} \frac{R_{l}^{l_{0}, m}(r)}{r} Y_{l, m}(\hat{\mathbf{r}}) .
\end{aligned}
$$

Since $m$ is a good quantum number, the Cartesian powers of the Gaussian functions (ie, $l_{\Gamma}, m_{\Gamma}$ and $n_{\Gamma}$ of Eq. (3)) can be restricted to sum to $m$. Substitution of this expansion into Eq. (16) leads to a set of complex linear equations for the unknown coefficients. If we choose the cutoff function $g(r)$ to be non-zero only for $r$-values beyond the range of the Gaussian functions, then no matrix elements between Gaussians and Coulomb functions are required in setting up the right hand side of the linear equations. The $T$-matrix elements can be obtained by matching the behavior of $\chi(\mathbf{r})^{l_{0} m}$ to the asymptotic form

$$
\chi(\mathbf{r})^{l_{0} m} \underset{r \rightarrow \infty}{\longrightarrow} \sum_{l} T_{l, l_{0}}^{m} Y_{l, m}(\hat{\mathbf{r}}) e^{i\left(k r+(Z / k) \ln 2 k r-\pi l / 2+\eta_{l}(k)\right)}
$$

at large values of $r$ where $g(r)$ is one.

In Tables III and IV we show elements of the lowest $3 \times 3$ block of the T-matrix computed using the hybrid basis using six real finite-elements of 17 th order DVR, and a complex turning point $R_{0}=50.0$ bohr. For these calculations, $l$-values up to 7 were included. A completely converged calculation would produce a complexsymmetric T-matrix. The near symmetry of the computed off-diagonal elements is an indication of the convergence of the computed results.

\section{Photoionization of $\mathbf{H}_{2}^{+}$}

In the weak-field limit, the $\mathrm{H}_{2}^{+}$photoionization amplitude is given by

$$
\left\langle\Phi^{(-)}(\mathbf{k}, \mathbf{r})|\epsilon \cdot \mathbf{r}| \Phi_{0}(\mathbf{r})\right\rangle
$$

where $\Phi_{0}(\mathbf{r})$ is the initial state target wave function, $\epsilon$ is the direction of polarization and the final continuum state $\Phi^{(-)}(\mathbf{k}, \mathbf{r})$ is related to $\Phi^{(+)}(\mathbf{k}, \mathbf{r})$ by

$$
\Phi^{(-)}(\mathbf{k}, \mathbf{r})=\left[\Phi^{(+)}(-\mathbf{k}, \mathbf{r})\right]^{*}
$$

Given the $\mathrm{H}_{2}^{+}$continuum functions and the initial state wave function, it is straightforward to evaluate the amplitude as a volume integral. However, it is also possible to derive a surface integral expression for the photoionization amplitude, which is easier to evaluate, as follows. We begin with the so-called first-order equation

$$
\left[E_{0}+\omega-H\right] \Psi_{\mathrm{sc}}=\epsilon \cdot \mathbf{r} \Psi_{0}
$$

where $E_{0}$ is the initial state energy and $\omega$ is the photon energy. Equation (21) can be solved, in complete analogy to Eq. (16), by expanding $\Psi_{\mathrm{sc}}$ in a combined Gaussian/DVR basis:

$$
\begin{aligned}
\Psi_{\mathrm{sc}} & =\sum_{\Gamma} c_{\Gamma} G_{\Gamma}(\mathbf{r})+\sum_{i, l} c_{i l} \frac{f_{i}(r)}{r} Y_{l, m}(\hat{\mathbf{r}}) \\
& \equiv \sum_{\Gamma} c_{\Gamma} G_{\Gamma}(\mathbf{r})+\sum_{l} \frac{\psi_{\mathrm{sc}}^{l, m}(r)}{r} Y_{l, m}(\hat{\mathbf{r}})
\end{aligned}
$$

and deriving complex linear equations for the expansion coefficients. 
TABLE III: $\mathrm{H}_{2}^{+}$T-matrix elements in ${ }^{2} \Sigma_{g}$ symmetry at $R=2.0$ bohr. Values of $l$ and $l^{\prime}$ refer to the incident and scattered angular momentum respectively.

\begin{tabular}{crcc}
\hline \hline & $l=0$ & $l=2$ & $l=4$ \\
\hline$k=0.632455$ & & & \\
$l^{\prime}=0$ & $-0.394697+0.806582 i$ & $0.010282-0.010774 i$ & $0.000060+0.000113 i$ \\
$l^{\prime}=2$ & $0.010253-0.010803 i$ & $0.287289+0.091121 i$ & $0.006738+0.002474 i$ \\
$l^{\prime}=4$ & $0.000061+0.000112 i$ & $0.006752+0.002420 i$ & $0.042134+0.001845 i$ \\
$\mathrm{k}=1.00000$ & & & \\
$l^{\prime}=0$ & $-0.396284+0.804174 i$ & $0.014883-0.014690 i$ & $0.000187+0.000429 i$ \\
$l^{\prime}=2$ & $0.014874-0.014701 i$ & $0.309300+0.108113 i$ & $0.016495+0.006679 i$ \\
$l^{\prime}=4$ & $0.000188+0.000428 i$ & $0.016505+0.006647 i$ & $0.049971+0.002882 i$ \\
$\mathrm{k}=1.41421$ & $-0.402412+0.796598 i$ & $0.007086-0.006476 i$ & $-0.000388+0.001282 i$ \\
$l^{\prime}=0$ & $0.007087-0.006478 i$ & $0.334214+0.129657 i$ & $0.029545+0.013468 i$ \\
$l^{\prime}=2$ & $-0.000387+0.001283 i$ & $0.029553+0.013448 i$ & $0.060234+0.004850 i$ \\
$l^{\prime}=4$
\end{tabular}

TABLE IV: $\mathrm{H}_{2}^{+}$T-matrix elements in ${ }^{2} \Sigma_{u}$ symmetry at $R=2.0$ bohr. Values of $l$ and $l^{\prime}$ refer to the incident and scattered angular momentum respectively.

\begin{tabular}{cccc}
\hline \hline & $l=1$ & $l=3$ & \multicolumn{1}{c}{$l=5$} \\
\hline $\mathrm{k}=0.632455$ & & & \\
$l^{\prime}=1$ & $0.385636+0.181877 i$ & $0.007367+0.004381 i$ & $-0.000004+0.000051 i$ \\
$l^{\prime}=3$ & $0.007388+0.004330 i$ & $0.091028+0.008465 i$ & $0.005503+0.000652 i$ \\
$l^{\prime}=5$ & $-0.000004+0.000051 i$ & $0.005502+0.000606 i$ & $0.023841+0.000618 i$ \\
$\mathrm{k}=1.00000$ & & & \\
$l^{\prime}=1$ & $0.327598+0.123193 i$ & $0.023564+0.011808 i$ & $0.000026+0.000333 i$ \\
$l^{\prime}=3$ & $0.023578+0.011771 i$ & $0.104795+0.011949 i$ & $0.011202+0.001536 i$ \\
$l^{\prime}=5$ & $0.000027+0.000333 i$ & $0.011202+0.001511 i$ & $0.029364+0.001028 i$ \\
$\mathrm{k}=1.41421$ & & & \\
$l^{\prime}=1$ & $0.239482+0.063844 i$ & $0.045646+0.018207 i$ & $0.000271+0.001023 i$ \\
$l^{\prime}=3$ & $0.045654+0.018183 i$ & $0.125938+0.018974 i$ & $0.018076+0.003034 i$ \\
$l^{\prime}=5$ & $0.000272+0.001022 i$ & $0.018078+0.003020 i$ & $0.036446+0.001742 i$ \\
\hline \hline
\end{tabular}


Figure 2 shows plots of the real parts of the $\Sigma_{u}$ and $\Pi_{u}$ components of $\Psi_{\mathrm{sc}}$ at $R=2.0 \mathrm{bohr}$ for the case of a $10 \mathrm{eV}$ photoelectron. The basis set parameters for these calculations were identical to those used in computing the $\mathrm{H}_{2}^{+}$continuum functions described above. The wave functions in these cases are plotted in a plane containing the molecular axis. It is interesting to note that the wave function is prominently directed along the axis of polarization; along the molecular axis in the case of parallel $\left(\Sigma_{u}\right)$ polarization and perpendicular to the molecular axis in $\Pi_{u}$ symmetry.

We can express the photoionization amplitude in terms of $\Psi_{\mathrm{sc}}$ by writing:

$$
\begin{aligned}
\left\langle\Phi^{(-)}(\mathbf{k}, \mathbf{r})|\epsilon \cdot \mathbf{r}| \Phi_{0}(\mathbf{r})\right\rangle & =\left\langle\Phi^{(-)}(\mathbf{k}, \mathbf{r})\left|(E-H)(E-H+i \epsilon)^{-1} \epsilon \cdot \mathbf{r}\right| \Phi_{0}(\mathbf{r})\right\rangle \\
& =\left\langle\Phi^{(-)}(\mathbf{k}, \mathbf{r})|E-H| \Psi_{\mathrm{sc}}(\mathbf{r})\right\rangle .
\end{aligned}
$$

The desired surface integral is obtained by allowing $H$ on the last line of Eq. (23) to operate to the left and using

Green's theorem to write:

$$
\text { the last line of Eq. (23) to operate to the left and using }
$$

$$
\begin{aligned}
\left\langle\Phi^{(-)}(\mathbf{k}, \mathbf{r})|\epsilon \cdot \mathbf{r}| \Phi_{0}(\mathbf{r})\right\rangle & =\left\langle\Phi^{(-)}(\mathbf{k}, \mathbf{r})|E-H| \Psi_{\mathrm{sc}}(\mathbf{r})\right\rangle \\
& =\frac{1}{2} \int_{S}\left[\Phi^{(-)^{*}}(\mathbf{k}, \mathbf{r}) \nabla \Psi_{\mathrm{sc}}-\Psi_{\mathrm{sc}} \nabla \Phi^{(-)^{*}}(\mathbf{k}, \mathbf{r})\right] \cdot d \mathbf{S} \\
& =\frac{1}{2} \sqrt{\frac{2}{\pi}} \sum_{l, m} i^{l} e^{i \eta_{l}(k)} Y_{l, m}(\hat{\mathbf{k}}) \\
\times & \left.\sum_{l^{\prime}}\left\{\left[\frac{\phi_{l, k}^{(\mathrm{c})}(r)}{k r} \delta_{l, l^{\prime}}+\frac{R_{l^{\prime}}^{l, m}(r)}{r}\right] \frac{d}{d r} \frac{\psi_{\mathrm{sc}}^{l^{\prime}, m}(r)}{r}-\frac{\psi_{\mathrm{sc}}^{l^{\prime}, m}(r)}{r} \frac{d}{d r}\left[\frac{\phi_{l, k}^{(\mathrm{c})}(r)}{k r} \delta_{l, l^{\prime}}+\frac{R_{l^{\prime}}^{l, m}(r)}{r}\right]\right\} r^{2}\right|_{r=S}
\end{aligned}
$$

Note that in deriving Eq. (24) we have assumed that the surface $S$ over which the integral is evaluated is large enough that the Gaussian functions are all zero, so that only the DVR contributions to $\Psi_{\text {sc }}$ and $\Phi^{(-)}(\mathbf{k}, \mathbf{r})$ are retained. The photoelectron momentum $\mathbf{k}$ and the polarization direction $\epsilon$ are conveniently expressed in a coordinate system tied to the molecular axis, which we denote by the vector $\hat{\mathbf{A}}$ in the laboratoiry frame. The differential photoionization cross section for an $\mathrm{H}_{2}^{+}$molecule with a specific fixed-in-space orientation is then given by

$$
\sigma(\mathbf{k}, \epsilon, \hat{\mathbf{A}})=4 \pi^{2} \frac{\omega k}{c}\left|\left\langle\Phi^{(-)}(\mathbf{k}, \mathbf{r})|\epsilon \cdot \mathbf{r}| \Phi_{0}(\mathbf{r})\right\rangle\right|^{2}
$$

For the total cross section, averaged over all molecular orientations, we can use the expression:

$$
\sigma_{\mathrm{tot}}=\frac{4 \pi \omega}{c} \operatorname{Im}\left\langle\Psi_{0}|\epsilon \cdot \mathbf{r}| \Psi_{\mathrm{sc}}\right\rangle
$$

The total photoionization cross sections for polarization parallel and perpendicular to the molecular axis are plotted in Figs. 3 and 4 , respectively. The agreement with the accurate numerical results of Bates and Opik [29] is essentially perfect.
The differential photoionization cross sections at $10 \mathrm{eV}$ photoelectron energy are shown in Fig. 5 for four different orientations of the molecule with respect to the direction of polarization. To our knowledge there are no other numerically accurate treatments with which to compare our results, so for comparison we show the unnormalized model results of Walter and Briggs [30], which were obtained using an ansatz " $2 \mathrm{C}$ " wave function. It is interesting to note that an accurate treatment shows that the photoelectron distribution follows the direction of photon polarization, while the simple $2 \mathrm{C}$ model incorrectly predicts alignment along the molecular axis.

\section{DISCUSSION}

Over the past five years or so, it has been convincingly demonstrated that grid-based methods are capable of fully solving problems in which two unbound electrons escape a simple atom or molecule. The principal difficulty with extending these purely numerical methods to anything but the simplest molecular targets centers on the need for a multi-center approach that avoids slowly 

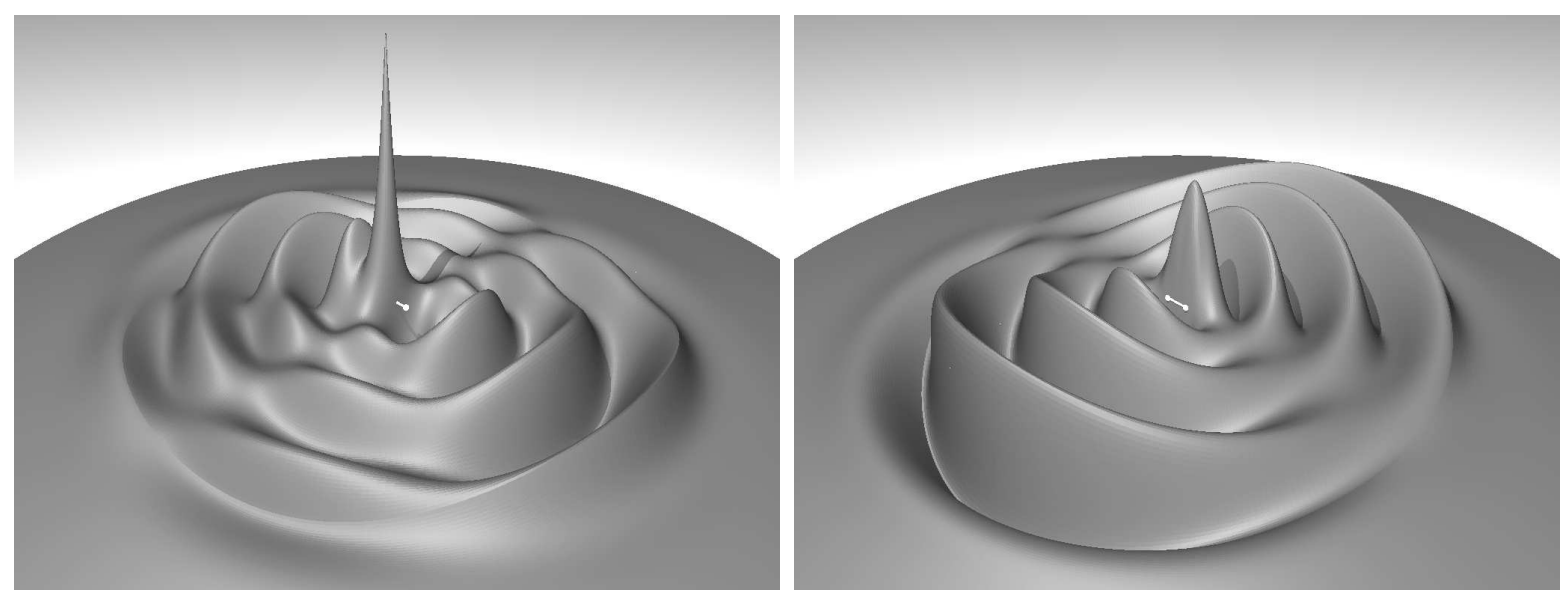

FIG. 2: Real part of scattered wave functions at $R=2.0$ bohr for $10 \mathrm{eV}$ photoelectrons in $\Sigma_{u}$ (left) and $\Pi_{u}$ (right) symmetry. The wave functions are plotted in a plane containing the molecular axis.

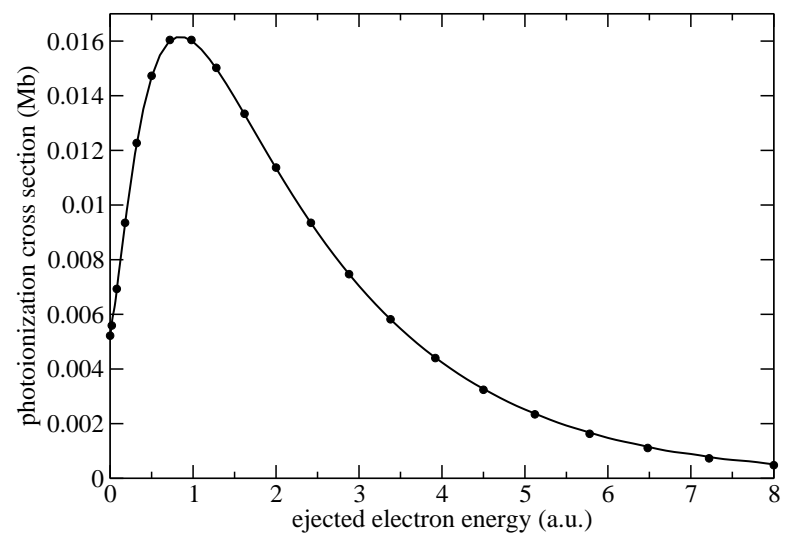

FIG. 3: Total photoionization cross section for polarization parallel to the molecular axis. Solid curve: current results; Points: Bates and Öpik [29].

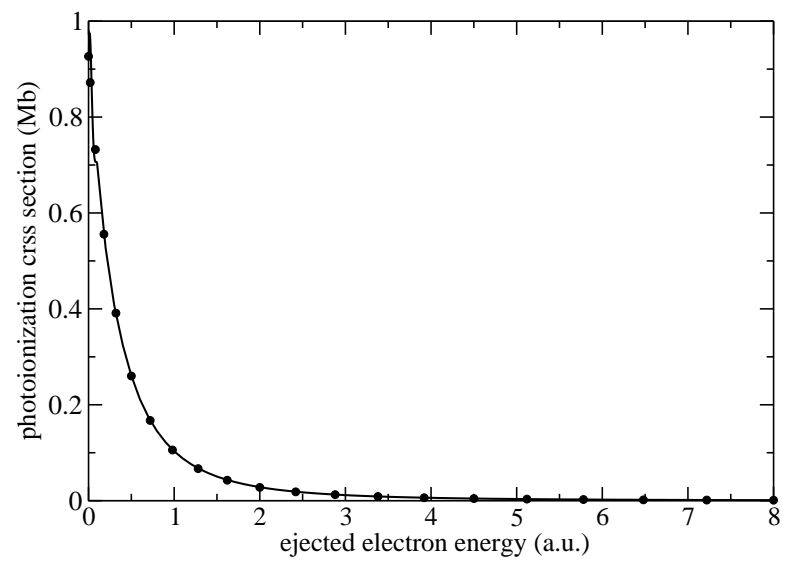

FIG. 4: Total photoionization cross section for polarization perpendicular to the molecular axis. Solid curve: current results; Points: Bates and Öpik [29].

convergent, single-center expansions, combined with an orbital description of inner-shell target electrons. We have argued that a practical connection with the techniques of quantum chemistry, which can readily address these difficulties, can be made by adopting a hybrid basis approach in which nuclear-centered Gaussian functions, which can span the physical regions of space where the target-electron charge density is significant, are combined with finite-element/DVR functions that do not penetrate the molecular core region but extend into the asymptotic domain. Exterior complex scaling of the outer DVR functions can then provide a basis for studying both bound and continuum problems. In this paper, we have taken the first steps toward demonstrating the viability of the approach by constructing such a basis and applying it to several one-electron molecular problems, namely, the bound states, continuum states and photoionization of $\mathrm{H}_{2}^{+}$. This method was indeed found to provide rapidly convergent results. Moreover, the required matrix elements could all be calculated using either analytic expressions or simple numerical quadrature. To go beyond the simple one-electron problems considered here, the critical step will be the demonstration that two-electron matrix elements can be rapidly and accurately evaluated. That demonstration, along with applications to a two-electron target molecule, will be the subject of a future publication.

\section{ACKNOWLEDGEMENTS}

This work was performed under the auspices of the US Department of Energy by the University of California Lawrence Berkeley National Laboratory under contract No. DE-AC02-05CH11231 and was supported by the U.S. DOE Office of Basic Energy Sciences, Division of Chemical Sciences. FLY acknowledges support from the National Science Foundation. 

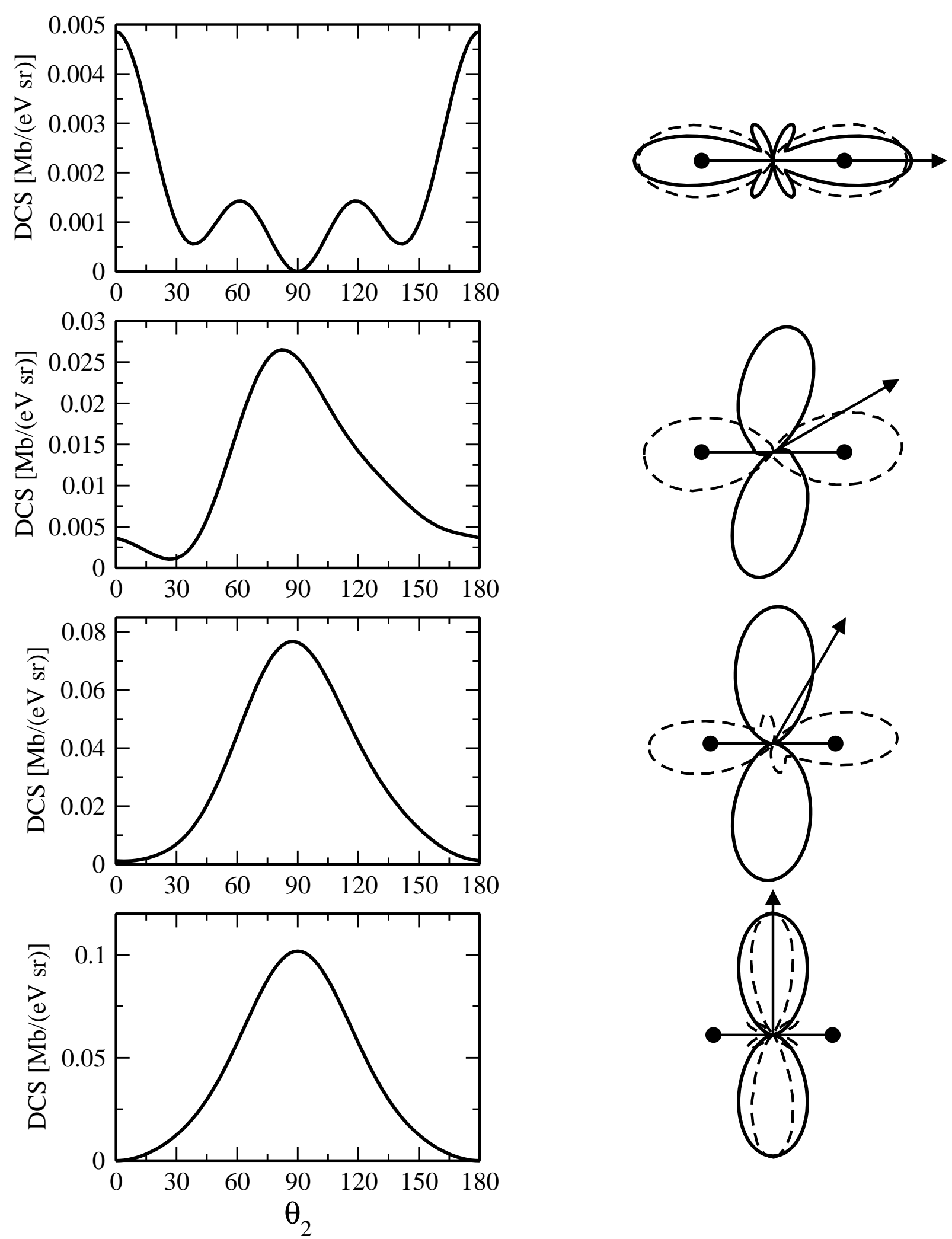

FIG. 5: Differential cross sections and angular distributions for $\mathrm{H}_{2}^{+}$at polarization angle $\theta=0^{\circ}, 30^{\circ}, 60^{\circ}$, and $90^{\circ}$ (top to bottom) with respect to the molecular axis (horizontal) and a $10.0 \mathrm{eV}$ ejected electron in the plane of the polarization and molecular axes. Solid curve: current result. Dashed curve: 2C un-normalized angular distributions of Walter and Briggs [30]. Note that for pure $\Sigma$ polarization (top panel), the cross sections are roughly twenty times smaller than for pure pure $\Pi$ polarization (bottom panel). 
[1] T.N. Rescigno, M. Baertschy, W.A. Isaacs, and C.W. McCurdy, Science 286, 2474 (1999).

[2] M. Baertschy, T.N. Rescigno, W.A. Isaacs, X. Li, and C.W. McCurdy, Phys. Rev. A 63, 022712 (2001).

[3] C.W. McCurdy, M. Baertschy and T.N. Rescigno, J. Phys. B 37, R137 (2004).

[4] W. Reinhardt, Ann. Rev. Phys. Chem. 33, 223 (1982).

[5] B. Simon, Phys. Lett. 71, 211 (1979).

[6] C.A. Nicolaides and D.R. Beck, Phys. Lett. A 65, 11 (1978).

[7] C.W. McCurdy and T.N. Rescigno, Phys. Rev. A 56, R4369 (1997).

[8] C.W. McCurdy, T.N. Rescigno and D. Byrum, Phys. Rev. A 56, 1958 (1997).

[9] T.N. Rescigno and C.W. McCurdy, Phys. Rev. A 62, 032706 (2000).

[10] C.W. McCurdy and F. Martin, J. Phys. B 37, 917 (2004).

[11] C.W. McCurdy, D.A. Horner, T.N. Rescigno and F. Martin, Phys. Rev. A 69, 032707 (2004).

[12] H. Bachau, E. Cormier, P. Decleva, J.E. Hansen and F. Martin, Rep. Prog. Phys. 64, 1815 (2001).

[13] B.I. Schneider, Phys. Rev. A 55, 3417 (1997).

[14] W. Vanroose, F. Martin, T.N. Rescigno and C.W. McCurdy, Phys. Rev. A 70, 050703 (R) (2004).

[15] T.N. Rescigno, M. Baertschy, D. Byrum and C.W. McCurdy, Phys. Rev. A 55, 4253 (1997).

[16] N. Rom, E. Engdahl, and N. Moiseyev, J. Chem. Phys.
93, 3413 (1990).

[17] N. Elander and E. Yarevsky, Phys. Rev. A 57, 3119 (1998).

[18] S.F. Boys, Proc. Roy. Soc. (London) A200, 542 (1950).

[19] R. McWeeny, Nature 166, 21 (1950).

[20] I. Shavitt, in Methods of Electronic Structure Theory, Ed. H.F. Schaefer III, Plenum Press, New York 189-275 (1977).

[21] S. Huzinaga, J. Chem. Phys. 42, 1293 (1965).

[22] W.J. Hehre, R.F. Stewart and J.A. Pople, J. Chem. Phys. 51, 2657 (1969).

[23] T.H. Dunning, Jr., J. Chem. Phys. 50, 2823 (1970).

[24] T.H. Dunning, Jr. and P.J. Hay, in Modern Theoretical Chemistry, vol.3, Ed. H.F. Schaefer III, Plenum Press, New York 1-28 (1977).

[25] R. Poirer, R. Kari and I.G. Czismadia, Handbook of Gaussian Basis Sets, Elsevier, Amsterdam (1985).

[26] D. E. Manolopoulos and R. E. Wyatt $C$ hem. Phys. Lett. 152, 23 (1988).

[27] V. I. Lebedev and D. N. Laikov, Doklady Mathematics 59, 477 (1999).

[28] M. M. Madsen and J. M. Peek, Atomic Data, 2, 171 (1971).

[29] D. R. Bates and U. Öpik, J. Phys. B 1, 543 (1968).

[30] M. Walter and J. Briggs, J. Phys. B 32, 2487 (1999). 